Document downloaded from:

http://hdl.handle.net/10251/112441

This paper must be cited as:

Fornés Leal, A.; Garcia-Pardo, C.; Castelló-Palacios, S.; Vallés Lluch, A.; Cardona Marcet, N. (207-). Accurate broadband measurement of electromagnetic tissue phantoms using open-ended coaxial systems. IEEE. 32-36. doi:10.1109/ISMICT.2017.7891761

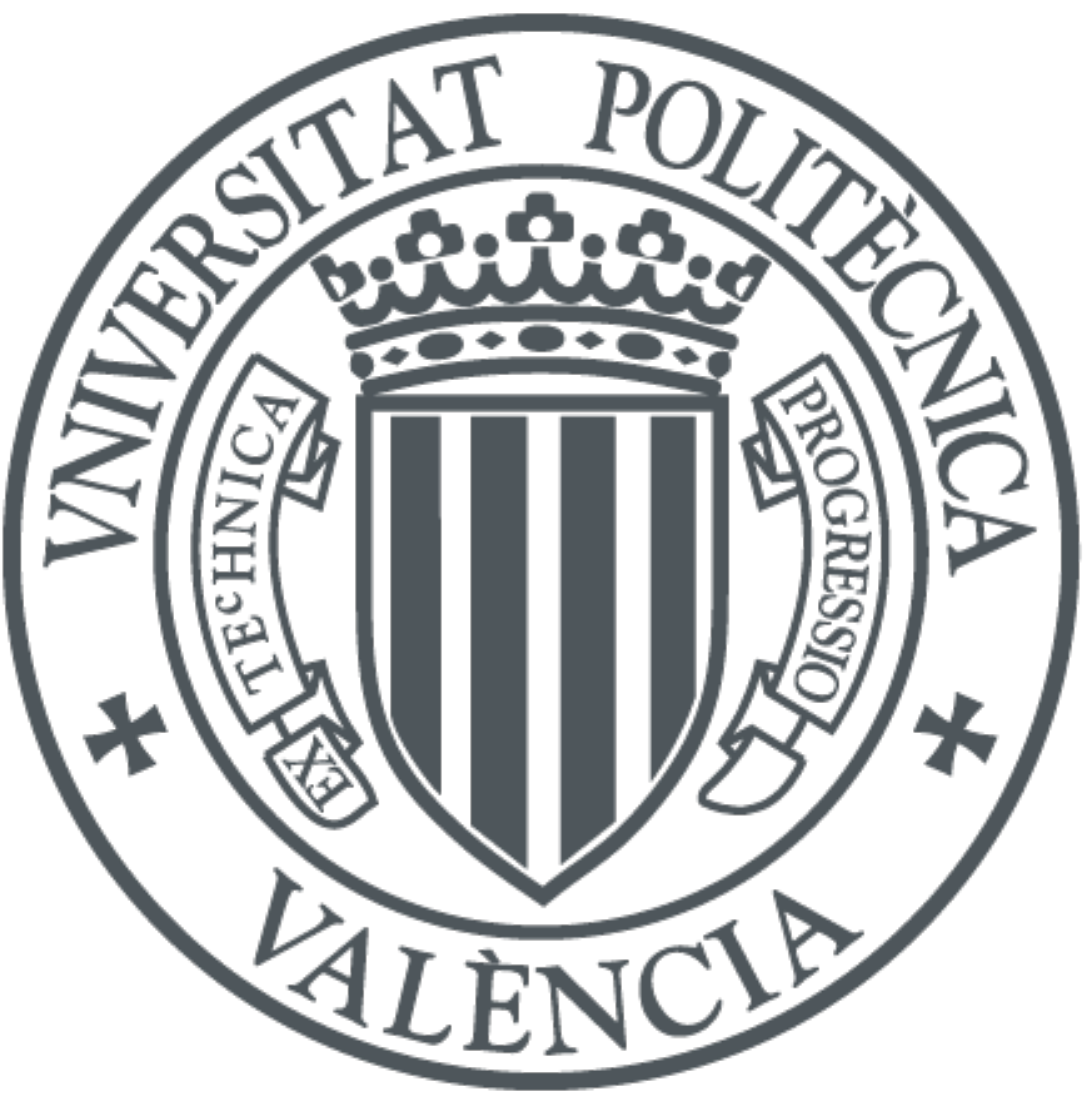

The final publication is available at

http://doi.org/10.1109/ISMICT.2017.7891761

Copyright IEEE

Additional Information 


\section{Accurate Broadband Measurement of Electromagnetic Tissue Phantoms Using Open-Ended Coaxial Systems}

\author{
Alejandro Fornes-Leal, Concepcion Garcia-Pardo, \\ Narcís Cardona \\ iTEAM \\ Universitat Politècnica de València \\ València, Spain \\ E-mail: alforlea@iteam.upv.es
}

\author{
Sergio Castelló-Palacios, Ana Vallés-Lluch \\ Centre for Biomaterials and Tissue Engineering \\ Universitat Politècnica de València \\ València, Spain \\ E-mail: sercaspa@etsii.upv.es
}

\begin{abstract}
New technologies and devices for wireless communication networks are continually developed. In order to assess their performance, they have to be tested in realistic environments taking into account the influence of the body in wireless communications. Thus, the development of phantoms, which are synthetic materials that can emulate accurately the electromagnetic behaviour of different tissues, is mandatory. An accurate dielectric measurement of these phantoms requires using a measurement method with a low uncertainty. The openended coaxial technique is the most spread technique but its accuracy is strongly conditioned by the calibration procedure. A typical calibration is performed using an open circuit, a short circuit and water. However, this basic calibration is not the most accurate approach for measuring all kinds of materials. In this paper, an uncertainty analysis of the calibration process of openended coaxial characterization systems when a polar liquid is added to the typical calibration is provided. Measurements are performed on electromagnetically well-known liquids in the 0.5 8.5 GHz band. Results show that adding methanol improves the accuracy in the whole solution domain of the system, mainly when measuring phantoms that mimic high water content tissues, whereas ethanol is more suitable for measuring low water content tissue phantoms.
\end{abstract}

Keywords- Calibration, electromagnetic phantoms, openended coaxial, permittivity measurement, probes.

\section{INTRODUCTION}

The development of new devices and technologies for wireless communication networks is growing fast over last decades. When the propagation of these wireless communications are influenced by the presence of body, they have to be evaluated carefully. To this end, it is necessary to develop accurate tissue phantoms, which are materials that imitates the electromagnetic characteristics of human tissues. They are cost-effective and easy to produce, allowing the assessment of different electromagnetic parameters related to transmission [1] and correlation [2] for wireless implanted devices in Body Area Networks (BAN), as well as specific absorption rate [3] and dosimetry in mobile communications scenarios. Electromagnetic tissue phantoms avoid as well the

This work was supported by the Ministerio de Educación y Ciencia, Spain (ref. TEC2014-60258-C2-1-R, TEC2014-56469-REDT), by the European FEDER funds. experimentation with animal specimens and humans in in vivo conditions [4] in preliminary stages.

The measurement method is a key factor to achieve a proper measurement of these phantoms. Thus, the open-ended coaxial method is widely used to characterize materials such as phantoms and tissues due to its many advantages. It is a nondestructive, easy to implement and low-cost solution that covers a large frequency range. This last is a key-point since it allows us to measure tissue phantoms in large bandwidths. The system captures the reflection coefficient of a wave applied to the measured material and processes it to obtain its permittivity.

Regarding the measurement procedure, calibration plays a crucial role since a properly transformation of the reflection coefficient requires a careful calibration. This is based on the measurement and the processing of the reflection coefficient of at least three different reference standards of well-known permittivity. Open circuit, short circuit and water are typically chosen as standards in the calibration (hereinafter "typical calibration") because their dielectric properties are very well characterized in literature. However, this calibration presents some limitations. Due to the very low dielectric constant of the open circuit standard and the high values of water and short circuit, the intermediate region is not properly characterized [5]. As a consequence, using these calibration standards provides a low loss factor accuracy above a few gigahertzs [6].

In order to reduce the uncertainty of the calibration process, some authors have suggested a variety of modifications, each of them with its strengths and weaknesses. One proposed solution is to use numerical techniques jointly with rigorous mathematical models to describe the probe [5], [7]. This solution provides a precise mapping to relate the reflection measurement and the permittivity when performing a typical calibration. However, it has a high computational cost and the real design parameters of the probe are needed. Other authors proposed the use of a matched load [8], but this approach has a difficult implementation due to the geometry of the sensor [9]. In [10] a short-cavity is used, but it has to be designed for a specific probe and perhaps fabricated by a third-party. 
The use of polar liquids in the calibration has also been suggested [11]. Non-polar liquids are discarded since their permittivities are very close to that of the open circuit. The strengths of this option are its non-dependence on the openended coaxial dimensions, and that it does not imply a huge effort in the redesign of the calibration process. Thus, this can be considered as the easiest solution to increase the accuracy of the system. Some authors have already proposed the use of methanol or saline solutions in addition to the typical standards or substituting some of them [12], [13].

However, to the best of our knowledge, there are no studies assessing how the accuracy related to the calibration process is modified depending on the calibration standards' choice. Hence, in order to evaluate the potential improvement that may entail using of polar liquids in the calibration, we present a comparison between the uncertainty of measurements when calibrating conventionally and with "enhanced" calibrations in which we add a polar liquid in the $0.5-8.5 \mathrm{GHz}$ frequency band. Measurements are made over electromagnetically wellknown materials whose permittivity is widely reported in literature. The accuracy of the measurement of body tissue phantoms can be improved if this liquid is added correctly.

This paper is organized as follows: the operation principle used for measuring the permittivity is presented in section II; the measurement system, the methodology and the analysis performed are described in section III; in section IV, the evaluation of different polar liquids as references in the calibration is presented. Results are discussed in section $\mathrm{V}$. Finally, conclusions are drawn in section VI.

\section{OPERATION PRINCIPLE}

The relative permittivity of a material, $\varepsilon_{r}$, is a complex frequency-dependent property determined by its response to an applied electromagnetic field [14]. Its real part $\varepsilon_{r}{ }^{\prime}$ is known as "dielectric constant" and its imaginary part $\varepsilon_{r}$ " is known as "loss factor".

$$
\varepsilon_{r}=\varepsilon_{r}^{\prime}-j \varepsilon_{r}^{\prime \prime}
$$

The electromagnetic characterization system consists of an open-ended coaxial line used as sensor and a computercontrolled vector network analyzer. It captures the reflection coefficient of the measured material and an external computer processes it in order to obtain a complex permittivity value. At the flat end of the sensor, the reflection coefficient $\Gamma_{m}$ is defined by the normalized admittance $y$ of the discontinuity as:

$$
\Gamma_{m}=\frac{1-y}{1+y}
$$

\section{A. Equivalent circuit model of the probe}

In order to model the normalize admittance $y$ of the discontinuity at the measurement plane, some equivalent circuit models and complete theoretical solutions have been presented in previous studies [5], [7], [15], [16]. The circuit model presented in [15] is used in this work as depicted in Figure 1. We have selected this model, since it is precise enough and does not involve an excessive complexity.

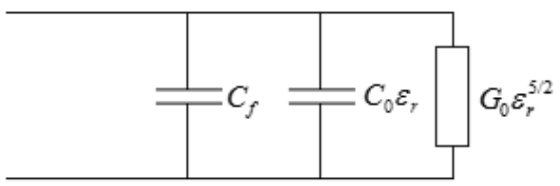

Fig. 1. Equivalent circuit model of the open-ended coaxial probe [15].

This model is formed by a free-space radiation conductance term, $G_{0} \varepsilon_{r}^{5 / 2}$, a capacitance term $C_{0} \varepsilon_{r}$ that denotes the fringing fields outside the coaxial cable, and another capacitance term, $C_{f}$, which represents the fringing field in the dielectric of the cable. Thus, this model relates the admittance of the probe's end with the permittivity $\varepsilon_{r}$ of the measured material by:

$$
y\left(\omega, \varepsilon_{r}\right)=G_{0} Z_{0} \varepsilon_{r}^{5 / 2}+j \omega Z_{0}\left(\varepsilon_{r} C_{0}+C_{f}\right)
$$

where $\omega$ is the angular frequency and $Z_{0}$ is the characteristic impedance of the probe. Combining (2) with (3), we obtain the relationship between the reflection coefficient $\Gamma_{m}$ and the relative permittivity $\varepsilon_{r}$ of the measured material as:

$$
\Gamma_{m}=\frac{1-G_{0} Z_{0} \varepsilon_{r}^{5 / 2}-j \omega Z_{0}\left(\varepsilon_{r} C_{0}+C_{f}\right)}{1+G_{0} Z_{0} \varepsilon_{r}^{5 / 2}+j \omega Z_{0}\left(\varepsilon_{r} C_{0}+C_{f}\right)}
$$

\section{B. Error correction procedure}

If we process directly the measured reflection coefficient $\rho_{m}$, it includes the errors that the instrumentation (e.g., cables, connectors) introduces due to its imperfections. These errors lead to a reduction of accuracy in the measurements. In order to reduce the effect of these imperfections, an error correction procedure is applied. It consists in modelling the elements between the reference plane (VNA's port) and the probe's end as a two-port network, also known as "error network". This concept has already been used in former studies [12], [13], and relates the measured reflection coefficient, $\rho_{m}$, to a corrected reflection coefficient, $\Gamma_{m}$, using the following transformation:

$$
\Gamma_{m}=\frac{\rho_{m}-S_{11}}{\rho_{m} S_{22}+S_{12} S_{21}-S_{11} S_{22}}
$$

where $\mathrm{S}_{x x}$ are the scattering parameters of the error network. Combining this equation with (2) and (3) and making some manipulations as shown in [17], the measured reflection $\rho_{m}$ can be related to the permittivity of the measured material by:

$$
\rho_{m}=\frac{A_{2}+A_{3}\left(\varepsilon_{r}+G_{n} \varepsilon_{r}^{5 / 2}\right)}{A_{1}+\varepsilon_{r}+G_{n} \varepsilon_{r}^{5 / 2}}
$$

where $A_{1}, A_{2}, A_{3}$ and $G_{n}$ are coefficients that depend on the frequency, the characteristic impedance of the probe, the parameters of the error network and the elements of the circuit model (Figure 1). These coefficients can be expressed as:

$$
\begin{gathered}
A_{1}=\frac{1-S_{22}}{\left(1+S_{22}\right) j \omega C_{0} Z_{0}}+\frac{C_{f}}{C_{0}} \\
A_{2}=\frac{S_{11}+S_{12} S_{21}-S_{11} S_{22}}{\left(1+S_{22}\right) j \omega C_{0} Z_{0}}+\frac{C_{f}}{C_{0}} \frac{\left(S_{11}-S_{12} S_{21}+S_{11} S_{22}\right)}{\left(1+S_{22}\right)}
\end{gathered}
$$




$$
\begin{gathered}
A_{3}=\frac{S_{11}-S_{12} S_{21}+S_{11} S_{22}}{1+S_{22}} \\
G_{n}=\frac{G_{0}}{j \omega C_{0}}
\end{gathered}
$$

The term $\mathrm{G}_{n}$ is usually neglected, especially when the sensor is not radiating into the measured material [16], [12]. However, we use (6) since it provides a more rigorous solution and allows us to introduce an extra standard in the calibration process.

\section{System calibration}

The unknown coefficients of (6) have to be computed measuring the reflection of four standards with well-known permittivities. After substituting these permittivities and their measured reflections into (6), a system of four equations with four unknowns has to be solved. Since permittivity depends on frequency, the system has to be solved for each frequency of interest. Once they are found, we will be able to transform a reflection measurement into its permittivity properly.

\section{MEASUREMENT SETUP AND METHODOLOGY}

\section{A. $\quad$ Measurement setup}

The setup used for the measurements consisted of a VNA (Keysight E5072A), an open-ended coaxial probe (Keysight 85070 E Slim Form Probe), a coaxial cable and the calibration standards. The VNA was controlled by an external computer via an USB interface and a MATLAB application.

The VNA was configured within the range of frequencies from 0.5 to $8.5 \mathrm{GHz}$, range where most of the applications for BAN and mobile communications work, with a power of 10 $\mathrm{dBm}, 801$ resolution points, and an IF bandwidth of $\mathrm{f}_{\mathrm{if}}=1 \mathrm{kHz}$.

\section{B. Methodology}

Three different liquids (hereinafter "tested samples") were measured using four different calibration setups: Ethylene Glycol, Dimethyl sulfoxide (DMSO) and 1-Butanol, whose permittivities are reported in [18]. Measurements were driven at a constant temperature of $25{ }^{\circ} \mathrm{C}$ since permittivity is a temperature-dependent property, checking that no air bubbles were present in the probe's end when measuring liquids. The calibration setups were:

- Typical calibration using open circuit and short circuit standards as well as distilled water.

- Three enhanced calibrations, which consist in the addition of one of the following polar liquids to the typical calibration: Methanol, Ethanol and 2-Propanol.

DMSO and 1-butanol have been chosen since they show an electromagnetic behaviour similar to tissues with high and low water content respectively. On the other hand, ethylene glycol allows us to observe how the accuracy increases when we use a liquid with similar behaviour in the calibration (methanol).

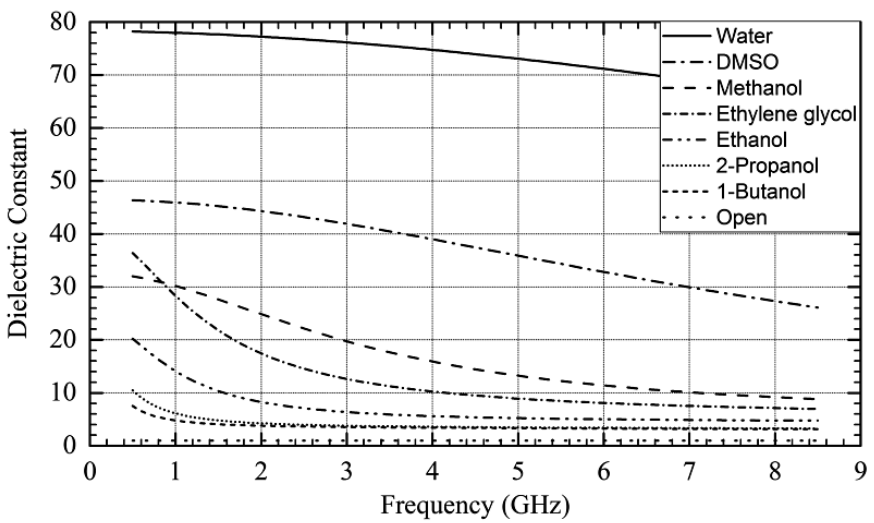

(a)

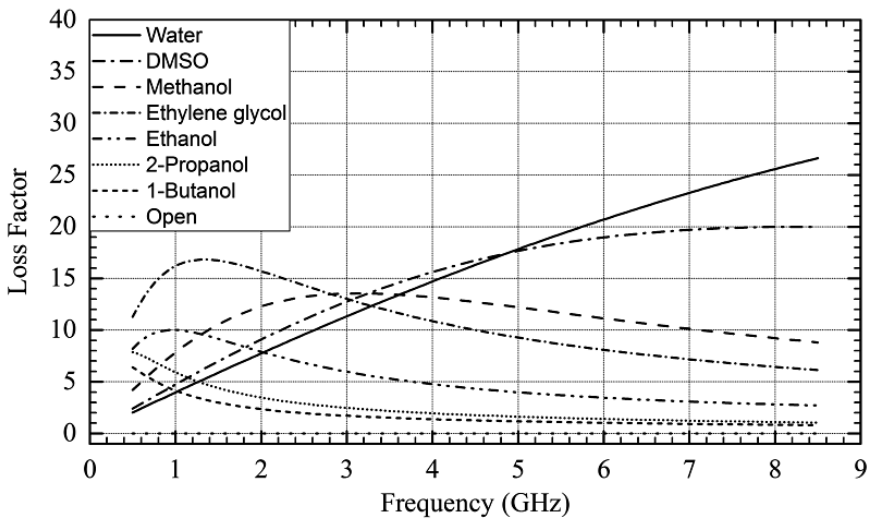

(b)

Fig. 2. Relative permittivity of calibration standards and tested samples at a temperature of $25^{\circ} \mathrm{C}$ : a) dielectric constant, b) loss factor.

The relative permittivity of the liquids used in this work are shown in Figure 2, taking the values from [18] since they have been characterized in a wide range of frequencies and temperatures using a method with a negligible uncertainty.

\section{Analysis of uncertainty}

Following the guidelines of [19], the sources of uncertainty of this kind of systems are:

- $\quad$ Random errors (Repeatability).

- Systematic errors: Calibration performance, VNA drifting with time, cable and system movements.

In this paper, only the systematic errors caused by the calibration process are evaluated. The repeatability and the drifting depend mainly on the VNA performance, and since they are independent of the polar liquids used in the calibration setup, they will not be object of study of this article. Cable movements will be neglected as well since the system will not be moved; the tested samples will be approached to the system instead. The systematic errors will be given in percentage terms, comparing the measurements of the tested samples with their theoretical values as:

$$
e^{\prime}=100 \times\left|\frac{\varepsilon_{m}^{\prime}-\varepsilon_{r e f}^{\prime}}{\varepsilon_{r e f}^{\prime}}\right|
$$

where $e^{\prime}$ are the systematic errors in dielectric constant, $\varepsilon^{\prime}{ }_{m}$ is 
the measured dielectric constant and $\varepsilon^{\prime}{ }_{r e f}$ is the theoretical one. The systematic errors in loss factor are computed analogously. In order to minimise the effect of random errors, five independent trials will be carried out, averaging the errors found for each calibration and for each tested sample.

\section{RESUltS}

The systematic errors of ethylene glycol's permittivity made by the different calibration setups are shown in Figure 3. One can observe that the lowest uncertainty is achieved by the enhanced calibration that uses methanol as the additional polar liquid, in both permittivity parts. This can be explained by the fact that the permittivities of methanol and ethylene glycol are similar in both real and imaginary parts, as it is seen in Figure 2. In contrast, compared to the results given by the typical calibration, the enhanced calibrations with 2-propanol and ethanol are only minimally useful at lower frequencies. In particular, ethanol enhanced calibration works better below 5 $\mathrm{GHz}$, whereas 2-propanol improves the measurement of the loss factor, comparing both with the typical calibration.

The systematic uncertainties of the measured permittivity of DMSO made by the different setups are shown in Figure 4. The results are similar to those obtained previously for ethylene glycol. Once again, adding methanol to the calibration process reduces the uncertainty with respect to the typical calibration while the other two polar liquids only achieve an improvement in loss factor. Calibrate with 2propanol and ethanol is not very useful in this case since their dielectric constants are very distinct compared to DMSO's.

In the same manner, the systematic errors of the measurements of 1-butanol's permittivity obtained by the different calibration setups are shown in Figure 5. In this case, all the enhanced calibrations have lower uncertainties than the typical one, being ethanol enhanced calibration the one that achieves the lowest uncertainty. However, in contrast to previous cases, methanol enhanced calibration reduces the uncertainty of the typical calibration in a lesser extent. Although the uncertainty seems quite high in this case, this is partially caused because the permittivity values that we are handling are very low, as it can be seen in Figure 2.

\section{DISCUSSION}

Analysing the uncertainty of the typical calibration in all cases, one can observe that it is higher in the imaginary part, and has an increasing trend above $5 \mathrm{GHz}$, corroborating the information presented in [6]. This occurs because the typical calibration does not provide a good mapping of the imaginary part, since open and short circuits have the same loss factor $\left(\varepsilon_{\mathrm{r}} "=0\right.$, Figure $\left.2 \mathrm{~b}\right)$. This becomes critical with the increment of frequency since water's loss factor increases with frequency and there is a big difference between them. In agreement with this, results showed that the addition of methanol improves the mapping of the loss factor for the whole solution domain. Since it also improves the mapping of the real part, we can establish that adding this polar liquid to the calibration process improves the accuracy when measuring any tissue phantom.

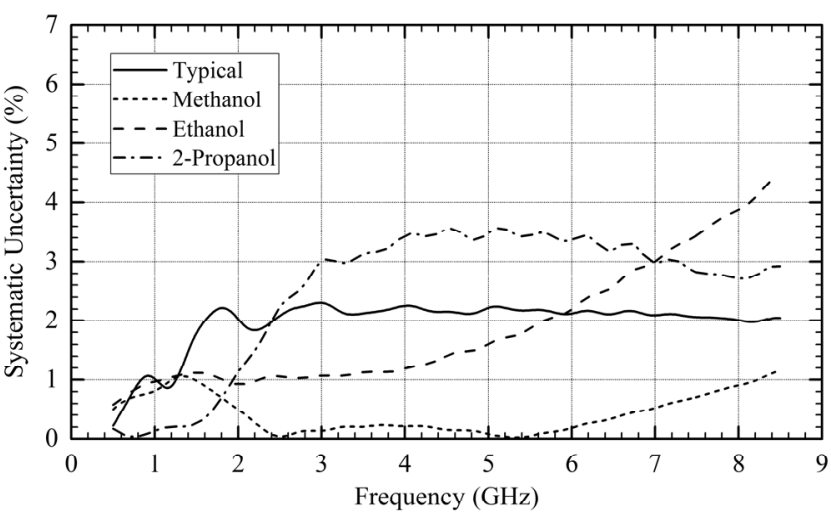

(a)

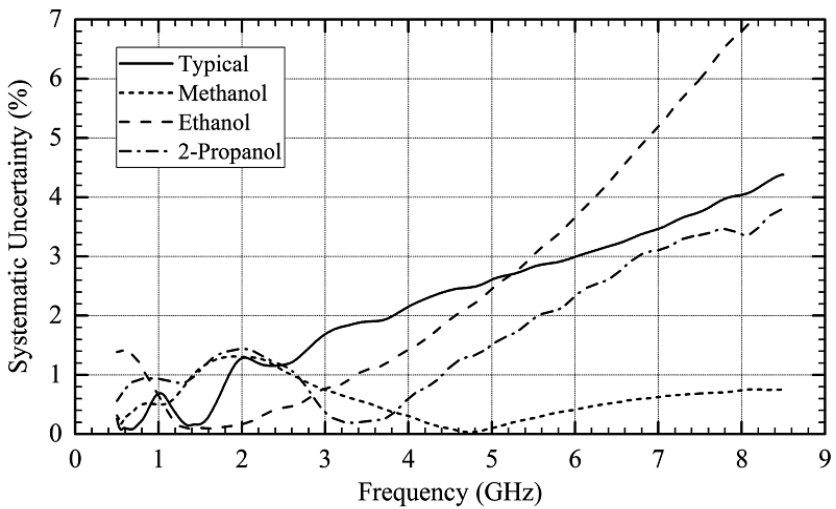

(b)

Fig. 3. Percentage of the systematic errors in the measurement of ethylene glycol with different calibration setups: a) dielectric constant, b) loss factor.

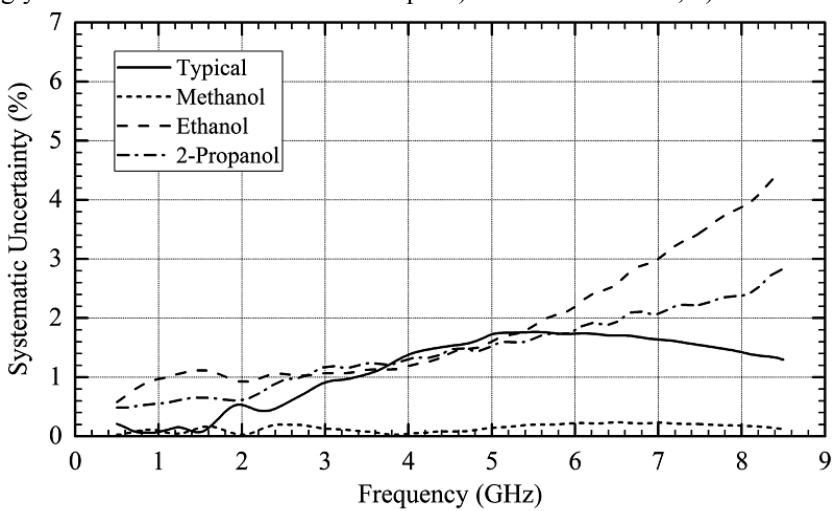

(a)

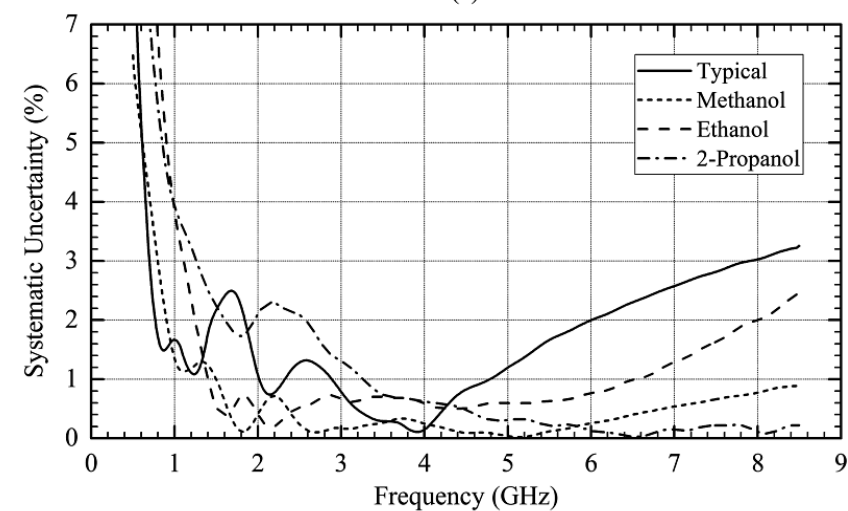

(b)

Fig. 4. Percentage of the systematic errors in the measurement of DMSO with different calibration setups: a) dielectric constant, b) loss factor. 


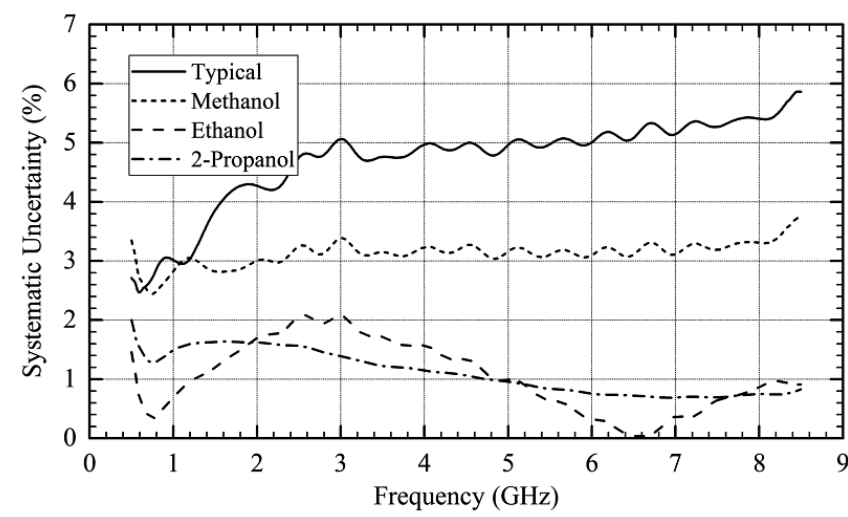

(a)

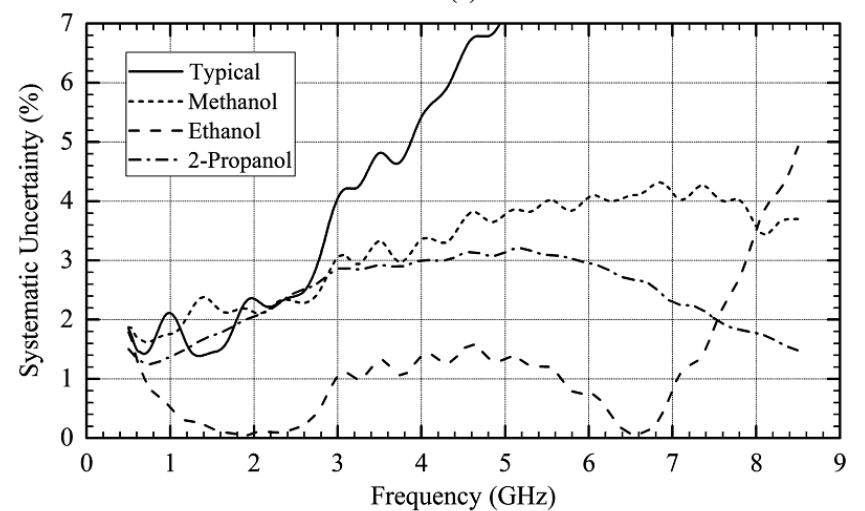

(b)

Fig. 5. Percentage of the systematic errors in the measurement of 1-butanol with different calibration setups: a) dielectric constant, b) loss factor.

We measured DMSO and 1-butanol since their dielectric properties are similar to low and high water content tissue phantoms. As a result, we can state that the addition of methanol in the calibration process is the best choice to measure high water content tissue phantoms, while ethanol is better to measure the low water content tissue phantoms.

\section{CONCLUSION}

In this paper, the addition of a polar liquid in the typical calibration of an open-ended coaxial system for measuring electromagnetic tissue phantoms is evaluated. To achieve this goal, the permittivities of ethylene glycol, dimethyl sulfoxide and 1-butanol were measured using four different calibration setups in the 0.5-8.5 GHz band. Measurements were conducted using the typical calibration (open circuit, short circuit and water), and repeated using enhanced calibrations by adding polar liquids such as methanol, ethanol and 2propanol. The systematic uncertainty of each calibration setup was evaluated comparing their measurements on testing materials with the reference values of these materials.

Results shown that when a liquid with dielectric properties similar to the material that is going to be measured is added to the typical calibration, a lower uncertainty of the system is always accomplished. Hence, adding methanol to the calibration improves the accuracy of the measurements of high water content tissue phantoms, whereas the addition of ethanol is more suitable for measuring low water content tissue phantoms. As future work, the uncertainty of adding methanol or other liquids in the calibration procedure of the system for measuring biological tissues should be evaluated since the properties of the measured material (heterogeneity, non-liquid) change.

\section{REFERENCES}

[1] R. Chavez-Santiago, C. Garcia-Pardo, A. Fornes-Leal, A. Valles-Lluch, G. Vermeeren, W. Joseph, I. Balasingham, and N. Cardona, "Experimental Path Loss Models for In-Body Communications within 2.36-2.5 GHz," IEEE J. Biomed. Heal. Informatics, pp. 1-1, 2015.

[2] C. Andreu, S. Castello-Palacios, C. Garcia-Pardo, A. Fornes-Leal, A. Valles-Lluch, and N. Cardona, "Spatial In-Body Channel Characterization Using an Accurate UWB Phantom," IEEE Trans. Microw. Theory Tech., vol. 64, no. 11, pp. 3995-4002, Nov. 2016.

[3] A. Y. Simba, S. Watanabe, T. Hikage, and T. Nojima, "Measurement of the maximum SAR in a spherical phantom when operating a mobile phone near a metallic wall," in 2008 First International Symposium on Applied Sciences on Biomedical and Communication Technologies, 2008, pp. 1-4.

[4] S. Castello-Palacios, C. Garcia-Pardo, A. Fornes-Leal, N. Cardona, and A. Valles-Lluch, "Tailor-Made Tissue Phantoms Based on Acetonitrile Solutions for Microwave Applications up to $18 \mathrm{GHz}$," IEEE Trans. Microw. Theory Tech., vol. 64, no. 11, pp. 3987-3994, Nov. 2016.

[5] D. V. Blackham and R. D. Pollard, "An improved technique for permittivity measurements using a coaxial probe," IEEE Trans. Instrum. Meas., vol. 46, no. 5, pp. 1093-1099, 1997.

[6] D. Misra, M. Chabbra, B. R. Epstein, M. Microtznik, and K. R. Foster, "Noninvasive electrical characterization of materials at microwave frequencies using an open-ended coaxial line: test of an improved calibration technique," IEEE Trans. Microw. Theory Tech., vol. 38, no. 1, pp. 8-14, 1990.

[7] J. R. Mosig, J.-C. E. Besson, M. Gex-Fabry, and F. E. Gardiol, "Reflection of an open-ended coaxial line and application to nondestructive measurement of materials," IEEE Trans. Instrum. Meas., vol. IM-30, no. 1, pp. 46-51, Mar. 1981.

[8] R. Zajicek and J. Vrba, "Broadband Complex Permittivity Determination for Biomedical Applications," in Advanced Microwave Circuits and Systems, InTech, 2010.

[9] J. S. Bobowski and T. Johnson, "Permittivity Measurements of Biological Samples By an Open-Ended Coaxial Line," Prog. Electromagn. Res. B, vol. 40, pp. 159-183, 2012.

[10] G. P. Otto and W. C. Chew, "Improved calibration of a large openended coaxial probe for dielectric measurements," IEEE Trans. Instrum. Meas., vol. 40, no. 4, pp. 742-746, 1991.

[11] A. Kraszewski, M. A. Stuchly, and S. S. Stuchly, "ANA Calibration Method for Measurements of Dielectric Properties," IEEE Trans. Instrum. Meas., vol. 32, no. 2, pp. 385-387, Jun. 1983.

[12] T. P. Marsland and S. Evans, "Dielectric measurements with an openended coaxial probe," IEE Proc. H Microwaves, Antennas Propag., vol. 134 , no. 4, pp. 341-349, 1987.

[13] A. Nyshadham, C. L. Sibbald, and S. S. Stuchly, "Permittivity measurements using open-ended sensors and reference liquid calibration-an uncertainty analysis," IEEE Trans. Microw. Theory Tech., vol. 40, no. 2, pp. 305-314, 1992.

[14] A. Wolski, "Theory of electromagnetic fields," Nov. 2011.

[15] M. A. Stuchly, M. M. Brady, S. S. Stuchly, and G. Gajda, "Equivalent circuit of an open-ended coaxial line in a lossy dielectric," IEEE Trans. Instrum. Meas., vol. IM-31, no. 2, pp. 116-119, Jun. 1982.

[16] M. A. Stuchly and S. S. Stuchly, "Coaxial Line Reflection Methods for Measuring Dielectric Properties of Biological Substances at Radio and Microwave Frequencies-A Review," IEEE Trans. Instrum. Meas., vol. 29, no. 3, pp. 176-183, 1980.

[17] J. Z. Bao, C. C. Davis, and M. L. Swicord, "Microwave dielectric measurements of erythrocyte suspensions.," Biophys. J., vol. 66, no. 6, pp. 2173-2180, 1994.

[18] A. P. Gregory and R. N. Clarke, "Tables of the Complex Permittivity of Dielectric Reference Liquids at Frequencies up to $5 \mathrm{GHz}$; NPL Report MAT 23," Innovation, no. March. NPL, pp. 1-87, 2009.

[19] A. Peyman, S. Holden, and C. G. Rum, "Dielectric Properties of Tissues at Microwave Frequencies," 2009. 